\title{
Gravitational instabilities in Kerr spacetimes
}

\author{
Gustavo Dotti ${ }^{1}$, Reinaldo J Gleiser ${ }^{1}$, Ignacio F Ranea-Sandoval ${ }^{2}$ and \\ Héctor Vucetich ${ }^{2}$ \\ ${ }^{1}$ Facultad de Matemática, Astronomía y Física (FaMAF), Universidad Nacional de Córdoba, \\ Ciudad Universitaria, (5000) Córdoba, Argentina \\ 2 Facultad de Ciencias Astronómicas y Geofísicas, Universidad Nacional de La Plata, \\ Paseo del Bosque S/N 1900, La Plata, Argentina
}

Received 5 September 2008

Published 2 December 2008

Online at stacks.iop.org/CQG/25/245012

\begin{abstract}
In this paper we consider the possible existence of unstable axisymmetric modes in Kerr spacetimes, resulting from exponentially growing solutions of the Teukolsky equation. We describe a transformation that casts the radial equation that results upon separation of variables in the Teukolsky equation, in the form of a Schrödinger equation, and combine the properties of the solutions of this equations with some recent results on the asymptotic behaviour of spin weighted spheroidal harmonics to prove the existence of an infinite family of unstable modes. Thus we prove that the stationary region beyond a Kerr black hole inner horizon is unstable under gravitational linear perturbations. We also prove that Kerr spacetime with angular momentum larger than its square mass, which has a naked singularity, is unstable.
\end{abstract}

PACS numbers: $04.50 .+\mathrm{h}, 04.20 .-\mathrm{q}, 04.70 .-\mathrm{s}, 04.30 .-\mathrm{w}$

\section{Introduction}

In 1916, shortly after Einstein published his vacuum field equations, Schwarzschild found a solution that represents what came to be known as a static black hole. It took almost 50 years to find a metric representing a rotating black hole. This is Kerr's solution [1], which, in Boyer-Lindquist coordinates reads

$$
\begin{aligned}
\mathrm{d} s^{2}=-\frac{\left(\Delta-a^{2} \sin ^{2} \theta\right)}{\Sigma} \mathrm{d} t^{2}-2 a \sin ^{2} \theta \frac{\left(r^{2}+a^{2}-\Delta\right)}{\Sigma} \mathrm{d} t \mathrm{~d} \phi \\
+\left[\frac{\left(r^{2}+a^{2}\right)^{2}-\Delta a^{2} \sin ^{2} \theta}{\Sigma}\right] \sin ^{2} \theta \mathrm{d} \phi^{2}+\frac{\Sigma}{\Delta} \mathrm{d} r^{2}+\Sigma \mathrm{d} \theta^{2},
\end{aligned}
$$

where $\Sigma=r^{2}+a^{2} \cos ^{2} \theta$ and $\Delta=r^{2}-2 M r+a^{2}$. Kerr's metric has two integration constants: the mass $M$, and the angular momentum per unit mass $a$. We will only consider the case $M>0$, and we will take $a \geqslant 0$ without loss of generality, since for $a<0$ we can 


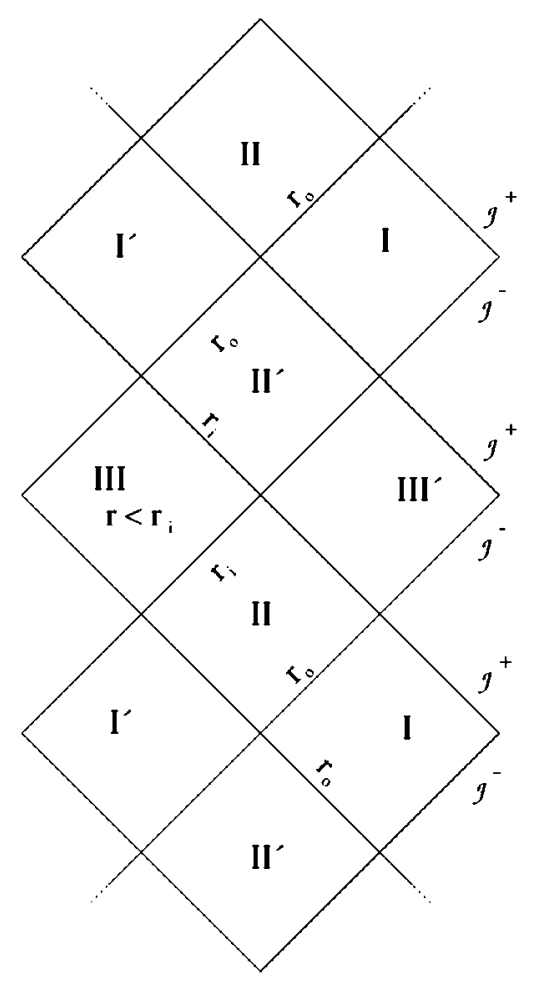

Figure 1. Penrose diagram for the maximal analytic extension of Kerr's spacetime. Regions labelled I and I' are isometric, and so are II and II', and III, III'

always change coordinates $\phi \rightarrow-\phi$, under which $a \rightarrow-a$. Setting $a=0$ (no rotation) gives Schwarzschild's solution. The $\Sigma=0$ ring singularity at $r=0, \theta=\pi / 2$ is hidden behind a black hole horizon as long as $a \leqslant M$. The inner and outer horizons are located at the zeroes of $\Delta: r_{i}=M-\sqrt{M^{2}-a^{2}}$ and $r_{o}=M+\sqrt{M^{2}-a^{2}}$, which are just coordinate singularities in (1). Kerr's spacetime can be extended through these horizons, and new regions isometric to $I: r>r_{o}, I I: r_{i}<r<r_{o}$ and III $: r<r_{i}$ arise ad infinitum, giving rise to the Penrose diagram displayed in figure 1 . According to the Carter-Robinson theorem and further results by Hawking and Wald, (see, e.g., [2] and references therein) if $\left(\mathcal{M}, g_{a b}\right)$ is an asymptoticallyflat stationary vacuum black hole that is non-singular on and outside its event horizon, then it must be a $0 \leqslant a<M$ member of the two-parameter Kerr family. In other words, the metric (1) with $a<M$ is the unique rotating black hole solution of Einstein's equations. All these features, together with its relevance to model astrophysical processes, signal Kerr's metric as one of the most important among the known exact solutions of Einstein's equations.

Regions I and III of a Kerr black hole are stationary, and finding out whether or not they are stable under gravitational perturbations is a fundamental issue. The stability of region I was established after a series of papers following Teukolsky's discovery of the perturbed fields admitting separable evolution equations [3], and ending with Whiting's proof that no solution of Teukolsky equations, supported in region I and properly decaying at infinity and $r_{o}$, can grow exponentially in time [4]. According to these results, small disturbances constrained to region I will oscillate around (1), in the same way a mechanical system oscillates around a local minimum configuration of its potential energy. Region III has a number of undesirable 
properties: it admits closed timelike curves, has the ring singularity, and lies beyond the Cauchy horizon, so that predictability is lost for earlier data. It has been argued [5] that a 'mass inflation' mechanism would prevent the formation of the Cauchy horizon, that would then be replaced by a null singularity acting as a boundary of the spacetime and cutting off this region. A fundamental issue to address is then the analysis of the linear stability of this region under gravitational perturbations. In this paper we show that region III is unstable by proving that there are solutions of Teukolsky equations, restricted to region III and satisfying appropriate boundary conditions, that grow exponentially in time. This instability of Kerr spacetime remained uncovered since Kerr's metric discovery 45 years ago and makes the analytic extension of Kerr's solution less relevant. One is immediately led to ask where region III is driven to by a gravitational perturbation that excites the unstable modes. Unfortunately, answering this question goes beyond the linear perturbation analysis in this paper.

We also prove in this paper that (1) with $a>M$ is unstable. For this 'super extremal' case, the ring singularity at $r=0, \theta=\pi / 2$ is naked, i.e., not protected by a black hole horizon, and can therefore causally connect with the rest of spacetime. The super extremal case was considered recently in [6], where numerical evidence for the existence of unstable modes was presented. Because of the nature of the numerical procedures used, the evidence was restricted to a range of values of $a / M$. Clearly, a more general result, establishing in a rigorous way the unstable nature of the Kerr spacetime in the case $a>M$ is of interest, as it suggests that such spacetimes cannot be the end point of the collapse of a self-gravitating system, a result of relevance in relation to the cosmic censorship conjecture. For other nakedly singular spacetimes such as the negative mass Schwarzschild solution and super extreme ReissnerNordström spacetimes, the linear instability was established after [7, 8] and [6, 9] respectively, where explicit expressions for unstable modes can be found. It should be stressed, however, that $a>M$ Kerr spacetimes are a subset of a larger family of axially symmetric solutions with a naked singularity, not singled out by the uniqueness theorems that make the black hole case so relevant.

This paper is organized as follows: section 2 reviews Teukolsky radial and angular equations, focusing on axisymmetric unstable modes. Although all we need from the angular equation is the behaviour of eigenvalues for small and large purely imaginary frequency, available in the recent literature [12], a technique to obtain approximations for eigenvalues and eigenfunctions is developed for completeness, and tested against the results in [12]. The reformulation of the radial Teukolsky equation that follows is crucial in this work. The radial equation is cast in Schrödinger-like form, and its potential analysed. This formulation is used in section 3 to prove that there exist infinitely many unstable modes for the super-extremal Kerr spacetime. Finally, in section 4, we adapt this proof to show that region III of a Kerr black hole is also unstable.

\section{Teukolsky equations}

The systematic analysis of the linearized perturbations of Kerr spacetime was greatly facilitated by Teukolsky's discovery [3] that, in the Newman-Penrose formalism, one can write second order partial differential evolution equations for two linearized null tetrad components $\Phi_{s}$ of the Weyl tensor, with $s= \pm 2$ the spin weight of these components. Furthermore these equations are separable. Introducing

$$
\Phi_{s}=R_{\omega, m, s}(r) S_{\omega, s}^{m}(\theta) \exp (\mathrm{i} m \phi) \exp (-\mathrm{i} \omega t),
$$

the linearized equations reduce to a coupled system for $S$ and $R$, 


$$
\begin{aligned}
& \frac{1}{\sin \theta} \frac{\mathrm{d}}{\mathrm{d} \theta}\left(\sin \theta \frac{\mathrm{d} S}{\mathrm{~d} \theta}\right)+\left(a^{2} \omega^{2} \cos ^{2} \theta-2 a \omega s \cos \theta-\frac{(m+s \cos \theta)^{2}}{\sin ^{2} \theta}+E-s^{2}\right) S=0 \\
& \Delta \frac{\mathrm{d}^{2} R}{\mathrm{~d} r^{2}}+(s+1) \frac{\mathrm{d} \Delta}{\mathrm{d} r} \frac{\mathrm{d} R}{\mathrm{~d} r}+\left\{\frac{K^{2}-2 \mathrm{i} s(r-M) K}{\Delta}\right. \\
& \left.+4 \mathrm{i} r \omega s-\left[E-2 a m \omega+a^{2} \omega^{2}-s(s+1)\right]\right\} R=0,
\end{aligned}
$$

where $\Delta=r^{2}-2 M r+a^{2}$, and $K=\left(r^{2}+a^{2}\right) \omega-a m$, with $M$ the mass, and $a$ the rotation parameter, related to the angular momentum $J$ by $J=a M$. Teukolsky's equations also apply to scalar $(s=0)$ and electromagnetic $(s= \pm 1)$ perturbations on the Kerr background. Moreover, any solution $\psi$ of the $s=-2$ Teukolsky equations is a 'Debye potential' from which a metric perturbation $h_{a b}$ that solves the linearized Einstein equations around the Kerr background can be constructed, $h_{a b}$ being obtained by applying a second order linear differential operator to $\psi$ (see [13]). Teukolsky's equations have been applied in a wide range of problems related to the behaviour of Kerr black holes under gravitational and other perturbations, including black hole collisions in the so-called 'close limit' [14]. A very important question addressed to from the early applications is that of the stability of region I of a Kerr black hole under gravitational perturbations, which was established through a series of works starting from [3] and ending with [4]. In what follows we will prove that region III of an $a<M$ Kerr spacetime, as well as the nakedly singular $a>M$ Kerr spacetimes, are unstable. This is done by showing that for these cases there exist solutions of the Teukolsky equations with purely imaginary frequency $\omega$ and satisfying appropriate boundary conditions. Our proof is based on an analysis of the behaviour of the lowest eigenvalues $E$ of (3) and (4) for large purely imaginary frequency $\omega$. Note that the coupling in the system (3)-(4) is rather subtle, and comes from this common eigenvalue $E$. Suppose $s$ and $m$ are fixed, then given $\omega, E$ has to be chosen so that $S$ is regular on the sphere (such a solution is called a spin weighted spheroidal harmonic, SWSH), and such that $R$ satisfies the required boundary conditions. The first condition gives a countable set of possible values $E_{\ell}(a \omega)$, whereas the second one fixes the possible $\omega$ values. Each one of these conditions can be cast in the form of a continued fraction equation [10] that arises when solving the three term recursion relation on the coefficients of series solution for $S$ and $R$ in (3) and (4).

\subsection{Spin weighted spheroidal harmonics}

For complex $\omega$ near $\omega=0$, a Taylor expansion for $E_{\ell}(a \omega)$ up to order $(a \omega)^{6}$ is available in the literature $([11,12]$ and references therein). Asymptotic expansions for $\omega \rightarrow \infty$ and for $\omega \rightarrow+\mathrm{i} \infty$ can be found in [12,15-17]. Particularly useful to our purposes is the fact that in the axial case $m=0$, and for gravitational perturbations $s= \pm 2$, the $E$ eigenvalue for large purely imaginary $\omega$ behaves as [12]

$$
\left.E_{\ell}(a \omega)\right|_{a \omega=\mathrm{i} k}=(2 \ell-3) k+\mathcal{O}\left(k^{0}\right), \quad \text { as } \quad k \rightarrow \infty .
$$

In the above formula it was assumed that that the $\ell$ labelling is chosen such that the $(\ell, m, s)$ SWSH reduces to the corresponding spin weighted spherical harmonic in the $a \omega=0$ limit [12], which implies

$$
\left.E_{\ell}(a \omega)\right|_{a \omega=0}=\ell(\ell+1),
$$

the relevant modes being $\ell=2,3,4, \ldots$. 
For the purposes of this paper we set $m=0, a \omega=\mathrm{i} k, s=-2$, and, after changing variables to $x=\cos \theta$ in (3), we find that the angular Teukolsky equation is

$$
\frac{\mathrm{d}}{\mathrm{d} x}\left[\left(1-x^{2}\right) \frac{\mathrm{d} S}{\mathrm{~d} x}\right]-\left(k^{2} x^{2}+\frac{4}{1-x^{2}}\right) S+\mathrm{i} 4 k x S=-E S .
$$

If $k=0$, (7) reduces to an associated Legendre equation, whose regular solutions are the associated Legendre polynomials $P_{\ell}^{(2)}(x), \ell=2,3, \ldots$, for which (6) holds. For real nonzero $k,(7)$ is a complex equation, but still the allowed $E$ values are real (see e.g., equation (2.3) in [12]). The symmetries of (7) for real $E$ imply that if $S(x)$ is a solution then so are $\overline{S(-x)}$ and $S_{ \pm}(x):=S(x) \pm \overline{S(-x)}$ (an overline denotes complex conjugation), so we can restrict our search to solutions with real and imaginary parts of opposite parity and, multiplying by $\mathrm{i}$ if necessary, we can always assume that $S(x)$ has even real and odd imaginary parts ${ }^{3}$ :

$$
S(x)=\overline{S(-x)} \text {. }
$$

Equation (7) has regular singular points at $x= \pm 1$. There is a regular Frobenius solution around $x=-1$ of the form $\mathcal{F}_{(-1)}(x)=\sum_{n \geqslant 1} c_{n}(E, k)(x+1)^{n}$, and a linearly independent solution diverging as $\ln (x+1)$. The series in the regular solution converges for $|x+1|<2$ [18], and is finite (in fact, vanishes) at $x=1$ if $E=\left.E_{\ell}(a \omega)\right|_{a \omega=\mathrm{i} k}$ for some $\ell$. Note that in this case $\mathcal{F}_{(-1)}(x)$ has to agree up to a multiplicative constant with the (regular) Frobenius series solution for (3) around $x=1$. A SWSH is a solution of (7) which is regular for $x \in[-1,1]$. Regularity in $[-1,1]$ will follow from regularity at, say, $x=-1$ if we impose the parity condition (8). Note, however, that since $S$ satisfies a second order ODE, the condition (8) is equivalent to $\Im(S(0))=\Re\left(S^{\prime}(0)\right)=0$. In particular, if $S=\left(\overline{\left.\mathcal{F}_{(-1)}(0)\right)} \mathcal{F}_{(-1)}(x)=\sum_{n, m \geqslant 1} c_{n}(E, k) \overline{c_{m}(E, k)}(x+1)^{n}\right.$, then $\Im(S(0))=0$, the solutions $E(k)$ of the equation

$0=\Re\left(S^{\prime}(0)\right)=(1 / 2) \sum_{n, m \geqslant 1} n\left(c_{n}(E, k) \overline{c_{m}(E, k)}+\overline{c_{n}(E, k)} c_{m}(E, k)\right)=: \mathcal{D}(E, k)$,

will equal $\left.E_{\ell}(a \omega)\right|_{a \omega=\mathrm{i} k}$ for some $\ell$, and $S$ will be regular for $x \in[-1,1]$ for these $E$ values.

As an application of this approach, we replaced $\mathcal{F}_{(-1)}(x)$ in $(9)$ with the regular Frobenius series at $x=-1$ up to order $(x+1)^{40}$, and numerically solved for the lowest $E$ (i.e., $\ell=2$ ) root in (9) for different values of $k$. The results, contrasted with the low $\omega$ expansion (see [11], [12] and references therein) and the asymptotic form (5) are shown in figure 2.

\subsection{Teukolsky radial equation, $a>M$ case}

Equation (4) is of the form $\Delta \ddot{R}+Q \dot{R}+Z R=0$, dots denoting derivatives with respect to $r$, and can be put in a suitable form for our analysis by introducing an integrating factor $L, \psi:=R / L$ and changing the radial variable to $r^{*}$, where $\frac{\mathrm{d} r^{*}}{\mathrm{~d} r}:=\frac{1}{f}$ with $f$ an unspecified positive definite function of $r$. The result is

$\frac{\Delta L}{f^{2}} \psi^{\prime \prime}+\left(\frac{2 L^{\prime} \Delta}{f^{2}}+\frac{Q L}{f}-\frac{\Delta L f^{\prime}}{f^{3}}\right) \psi^{\prime}+\left(\frac{\Delta L^{\prime \prime}}{f^{2}}+\frac{Q L^{\prime}}{f}-\frac{\Delta L^{\prime} f^{\prime}}{f^{3}}+Z L\right) \psi=0$

where' denotes derivatives with respect to $r^{*}$. The choice $f=\sqrt{\Delta}$ (recall $\Delta$ is strictly positive in the super extreme case $a>M$ ) gives an adimensional $r^{*}$,

$r^{*}=\ln \left(\frac{r-M+\sqrt{r^{2}-2 M r+a^{2}}}{M}\right) \simeq \begin{cases}\ln \left(\frac{2 r}{M}\right) & r \rightarrow \infty \\ \ln \left(\frac{a^{2}-M^{2}}{2 M|r|}\right) & r \rightarrow-\infty\end{cases}$

${ }^{3}$ Note that, with this overall phase choice, in the $k \rightarrow 0$ limit $S$ will reduce to a real multiple of $P_{\ell}^{(2)}\left(\mathrm{i} P_{\ell}^{(2)}\right)$ if $\ell$ is even (odd). Note also that our phase choice is different from the choices in $[6,12]$. 

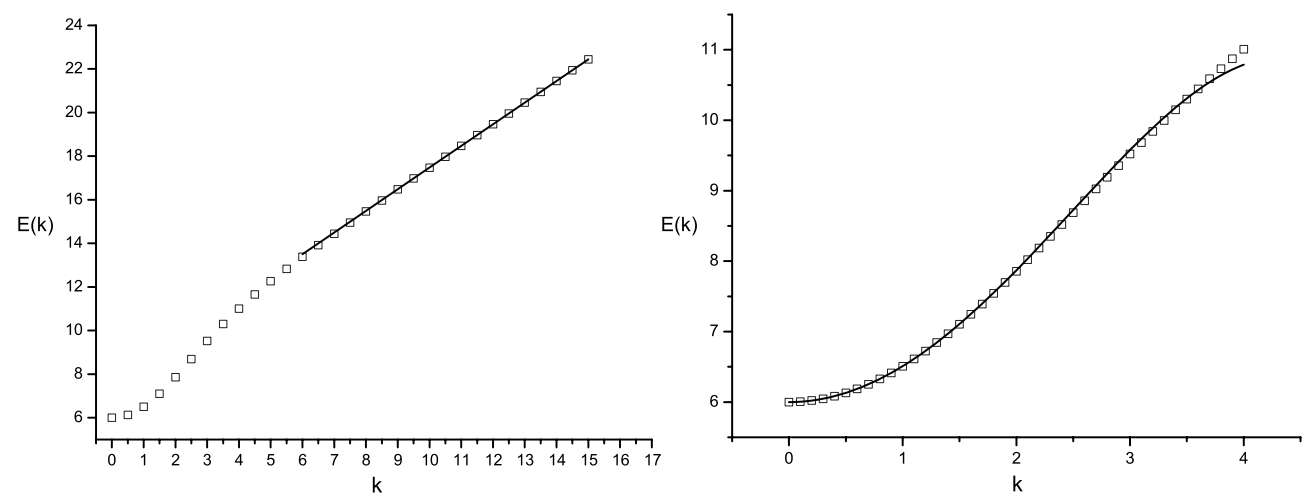

Figure 2. The left panel shows the lowest (corresponding to $\ell=2$ ) $E$ roots obtained by solving equation (9) for 30 different values of $k \in[0,15]$, and a least square linear fit for the ten points in the interval $10 \leqslant k \leqslant 15$, which gave $E=0.993 k+$ constant, in excellent agreement with the expected asymptotic expansion in equation (5). The right panel shows 40 values obtained in this way for $0<k<4$ (dots) and the low frequency approximation in [11,12] up to order $k^{4}$ (solid line).

that grows monotonically with $r$, and can easily be inverted in terms of elementary functions,

$$
r=\frac{M \exp \left(r^{*}\right)}{2}+M+\frac{M^{2}-a^{2}}{2 M \exp \left(r^{*}\right)}
$$

The next step is to choose $L$ such that the $\psi^{\prime}$ coefficient of (10) vanishes. This gives $L=\Delta^{-\frac{(2 s+1)}{4}}$ with (4) reducing to a Schrödinger-like equation

$$
\mathcal{H} \psi:=-\psi^{\prime \prime}+V \psi=-E \psi
$$

The potential

$$
V:=-\left(\frac{\Delta \ddot{L}}{L}+\frac{Q \dot{L}}{L}+Z+E\right)
$$

is independent of $E$ (recall that $Z$ is the coefficient of $R$ in (4)), this being the reason behind the above choice of $r^{*}$. For $s=-2, m=0$, and $\omega=\mathrm{i} k / a$, the case we are interested in, the potential is real and reads

$$
\begin{array}{r}
V=\left[\frac{r^{4}+a^{2} r^{2}+2 a^{2} M r}{a^{2}\left(r^{2}-2 M r+a^{2}\right)}\right] k^{2}+4\left[\frac{-r^{3}+3 M r^{2}-a^{2} r-a^{2} M}{a\left(r^{2}-2 M r+a^{2}\right)}\right] k \\
+\left[\frac{r^{2}-2 M r+15 M^{2}-14 a^{2}}{4\left(r^{2}-2 M r+a^{2}\right)}\right]=: k^{2} V_{2}+k V_{1}+V_{o} .
\end{array}
$$

Since $a>M$ in the super-extreme case, $V$ is smooth everywhere. Also, $V$ is bounded from below for every $k \geqslant 0$, its minimum being not continuous, as a function of $k$, at $k=0$ :

$$
\begin{aligned}
& \min \{V(r, k=0), r \in \mathbb{R}\}=-7 / 2 \\
& \lim _{k \rightarrow 0^{+}} \min \{V(r, k), r \in \mathbb{R}\}=-15 / 4 .
\end{aligned}
$$




\section{Unstable modes of the Kerr naked singularity}

$\mathcal{H}$ in (13) is self-adjoint in the Hilbert space of square integrable functions of $r^{*}$ with the Hermitian product $\langle\alpha \mid \beta\rangle:=\int \bar{\alpha} \beta \mathrm{d} r^{*}$. Given that $V$ is smooth, bounded from below, and

$$
V \sim\left(\frac{M k e^{r^{*}}}{2 a}\right)^{2}, \quad\left|r^{*}\right| \rightarrow \infty,
$$

the spectrum of the self-adjoint operator $\mathcal{H}$ is fully discrete and has a lower bound. A careful analysis of (11), (15) and (17), or, more directly, the asymptotic form of (4), indicates that the square integrable eigenfunctions of $\mathcal{H}$ behave as

$$
\psi \sim \begin{cases}\mathrm{e}^{-\frac{r k}{a}}\left(\frac{M}{r}\right)^{\frac{2 k M}{a}-3}(1+\mathcal{O}(M / r)), & r \rightarrow \infty \\ \mathrm{e}^{\frac{r k}{a}}\left(\frac{M}{r}\right)^{1-\frac{2 k M}{a}}(1+\mathcal{O}(M / r)), & r \rightarrow-\infty\end{cases}
$$

with sub-leading terms that depend on the eigenvalue.

To obtain some useful information about the fundamental energy of the radial Hamiltonian (13), we need to analyse its potential (15). The numerator of $V_{2}, r\left(r^{3}+a^{2} r+2 a^{2} M\right)$, is negative in some interval to the left of zero, and, since $V_{1}(0)=-4 a^{2} M<0$, by continuity $V_{1}$ must also be negative in some neighbourhood of $r=0$. Thus, there is an interval $s_{1}(M)<r<s_{2}(M)<0$ where both $V_{1}$ and $V_{2}$ are negative. Let $\psi$ be a smooth normalized $(\langle\psi \mid \psi\rangle=1)$ test function supported on $r \in\left(s_{1}(M), s_{2}(M)\right)$. Note that $\psi$ has compact support away from $r=0$, and that

$$
\langle\psi|\mathcal{H}| \psi\rangle=\left\langle\psi\left|-\left(\partial / \partial r^{*}\right)^{2}\right| \psi\right\rangle+\sum_{j=0}^{2} k^{j}\left\langle\psi\left|V_{j}\right| \psi\right\rangle
$$

with

$$
\left\langle\psi\left|V_{j}\right| \psi\right\rangle=\int_{s_{1}(M)}^{s_{2}(M)}|\psi|^{2} V_{j} \frac{\mathrm{d} r}{\sqrt{\Delta}}<0 \quad \text { for } \quad j=1,2 .
$$

Since $\left\langle\psi\left|V_{1}\right| \psi\right\rangle$ is negative, there is a $k_{c}$ such that, for $k>k_{c},\left\langle\psi\left|-\left(\partial / \partial r^{*}\right)^{2}\right| \psi\right\rangle+\left\langle\psi\left|V_{0}\right| \psi\right\rangle+$ $k\left\langle\psi\left|V_{1}\right| \psi\right\rangle$ is negative and so $\langle\psi|\mathcal{H}| \psi\rangle<k^{2}\left\langle\psi\left|V_{2}\right| \psi\right\rangle$. This implies that if $-\epsilon_{o}(k)$ is the lowest eigenvalue of $\mathcal{H}$ then

$$
-\epsilon_{o}(k) \leqslant\langle\psi|\mathcal{H}| \psi\rangle<k^{2}\left\langle\psi\left|V_{2}\right| \psi\right\rangle<0, \quad \text { if } \quad k>k_{c} .
$$

From (16) and the above equation

$$
\epsilon_{o}\left(k=0^{+}\right)<\frac{15}{4}, \quad \epsilon_{o}(k)>\left|\left\langle\psi\left|V_{2}\right| \psi\right\rangle\right| k^{2}, \quad k>k_{c} .
$$

From (5), (6) and (22) it is clear that, for any $\ell=2,3, \ldots$, the curves $\epsilon_{o}(k)$ and $\left.E_{\ell}(a \omega)\right|_{a \omega=\mathrm{i} k}$ intersect at some $k_{\ell}>0$. We have numerically checked this behaviour and spotted the predicted intersection point $k_{(\ell=2)}$. The results, shown in figure 3, are in perfect agreement with those in [6].

This implies that there is a common eigenvalue $E=\left.E_{\ell}(a \omega)\right|_{a \omega=\mathrm{i} k_{\ell}}=\epsilon_{o}\left(k_{\ell}\right)$ for the radial and angular Teukolsky equations and that $\left\{S(\theta)=S_{\left(\ell, m=0, k_{o}\right)}(\theta), R(r)=\Delta^{\frac{3}{4}} \psi_{o}^{\left(k_{\ell}\right)}(r)\right\}, \ell=$ $2,3, \ldots$ are (infinitely many!) solutions of the Teukolsky equations, all of which correspond to unstable gravitational perturbations $\Phi_{(s=-2)}(t, r, \theta)=S_{\left(\ell, m=0, k_{o}\right)}(\theta) \Delta^{\frac{3}{4}} \psi_{o}^{\left(k_{\ell}\right)}(r) \exp \left(k_{\ell} t / a\right)$ with $\psi_{o}^{\left(k_{\ell}\right)}(r)$ behaving as in (18). The radial exponential decay rate guarantees that any quantity of interest vanishes asymptotically for large $r$. 


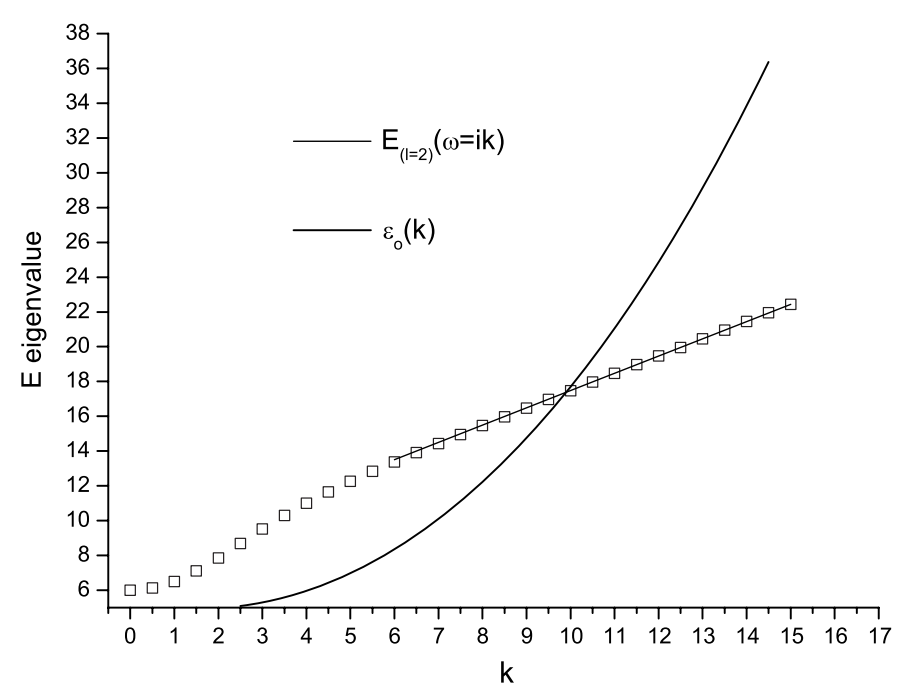

Figure 3. Intersection of the numerically generated curves $\left.E_{(\ell=2)}(a \omega)\right|_{a \omega=\mathrm{i} k}$ and $\epsilon_{o}(k)$ for $a / M=1.4$. Note the agreement with the results in [6], according to which for $a=1.4$ the intersection should occur at $k \simeq 7.07 \times 1.4=9,898$.

Regarding the behaviour of the perturbation at the ring singularity we point out that: (i) contrary to what happens for other naked singularities (e.g., the negative mass Schwarzschild solution, see [7,8]), $r=0$ is not a singular point of the radial equation, the potential (15) being smooth everywhere. In fact, $r=0$ is neither a boundary point, nor a singular point of the second order radial equation (13), then imposing conditions at this point, besides requiring that the perturbations vanishes at $r^{*}= \pm \infty$, would lead to an unnaturally over-determined problem. (ii) It is natural to ask, however, what is the effect of the perturbation on the singularity? In particular, one wants to make sure that first order corrections to the Riemann tensor invariants do not diverge faster than the zeroth-order term as we approach the singularity, otherwise, the use of linearized equations is not justified, since we cannot keep the perturbation small in the whole spacetime. As shown in [6], no algebraic invariant gets corrections under our unstable modes. A deeper treatment of the perturbed geometry could possibly be afforded in the approach where the $s=-2$ solutions of the Teukolsky equation are used as 'potentials' to construct metric perturbations [13], but this is a rather technical analysis [19] that certainly exceeds the scope of this report.

Note that the unstable perturbations are not restricted to the fundamental radial mode, since for large $k$, the potential $V$ in (13) has a deep minimum that can be approximated by a harmonic oscillator potential of depth of order $k^{2}$, and strength also of order $k^{2}$, so that the level spacing near the ground state of $\mathcal{H}$ is of order $k$. Thus, the negative $-\epsilon_{n}(k)$ of the lowest radial eigenvalues also grow quadratically in $k$ for large $k$, and intersect the angular eigenvalue curves for sufficiently large values of $k$ [19].

\section{Unstable modes for region III of a Kerr black hole}

The calculations above can be easily adapted to deal with perturbations in the interior region $r<r_{i}:=M-\sqrt{M^{2}-a^{2}}$ of an $a \leqslant M$ Kerr black hole. The extreme $a=M$ and sub-extreme $a<M$ cases require separate treatments. 


\subsection{Extreme case $(a=M)$ inner region}

The solution of $\mathrm{d} r^{*} / \mathrm{d} r=1 / \sqrt{\Delta}$ in the interior region $r<r_{i}=r_{o}=M$ is

$$
r^{*}=-\ln \left(\frac{M-r}{M}\right), \quad r<r_{i}
$$

with inverse

$$
r=M\left(1-\mathrm{e}^{-r^{*}}\right), \quad-\infty<r^{*}<\infty .
$$

Using the integration factor $\Delta^{3 / 4}$ as before, we are led back to (13) and (15), with $r$ given in (24). Note that

$$
V \sim \begin{cases}4 k^{2} \exp \left(2 r^{*}\right), & r^{*} \rightarrow \infty \\ k^{2} \exp \left(-2 r^{*}\right), & r^{*} \rightarrow-\infty\end{cases}
$$

then the spectrum of the self-adjoint operator $\mathcal{H}$ is again fully discrete and has a lower bound. The eigenfunctions behave as

$\psi \sim \begin{cases}\left(\frac{M}{M-r}\right)^{2 k} \exp \left[-2 k\left(\frac{M}{M-r}\right)\right]\left(1+\mathcal{O}\left(\frac{M-r}{r}\right)\right), & r \rightarrow M^{-} \\ \frac{M^{1-2 k}}{r} \mathrm{e}^{\frac{r k}{M}}(1+\mathcal{O}(M / r)), & r \rightarrow-\infty .\end{cases}$

The argument of instability in the super extreme case goes through in this case without modifications, since the test function in (19) is supported in the $r<0$ region, and thus can be used again in this case to obtain the bound (21). The radial decay (26) guarantees that corrections to relevant quantities vanish in these limits. The considerations about the perturbation behaviour near the ring singularity for the super extremal case apply again to this case, and also to the sub-extreme case below.

\subsection{Sub-extreme case $(a<M)$ inner region}

The sub-extreme case introduces some subtleties: requiring $\mathrm{d} r^{*} / \mathrm{d} r=1 / \sqrt{\Delta}$ for $r<r_{i}$ gives

$r^{*}=\ln \left(\frac{M-r-\sqrt{r^{2}-2 M r+a^{2}}}{M}\right)=\ln \left(\frac{r_{i}+r_{o}-2 r-2 \sqrt{\left(r_{o}-r\right)\left(r_{i}-r\right)}}{r_{i}+r_{o}}\right)$,

$$
r<r_{i}
$$

and the radial equation reduces to (13), (15) with $r$ the inverse of (27).

Since

$$
-\infty<r^{*}<r_{i}^{*}:=\ln \left(\frac{r_{o}-r_{i}}{r_{o}+r_{i}}\right),
$$

(13) is, in this case, a Schrödinger-like equation on a half-axis, with potential diverging as

$$
V \sim\left[k\left(M^{2}-a^{2}\right) /(2 a M)\right]^{2} \exp \left(-2 r^{*}\right)
$$

for $r^{*} \rightarrow-\infty$ and, for $r^{*} \rightarrow r_{i}^{*-}$, as

$$
\begin{gathered}
V \sim\left[-\frac{1}{4}+\frac{4 M^{2}\left(M-\sqrt{M^{2}-a^{2}}\right)^{2}}{M^{2}-a^{2}}\left(\frac{k}{a}-\frac{\sqrt{M^{2}-a^{2}}}{M\left(M-\sqrt{M^{2}-a^{2}}\right)}\right)^{2}\right] \\
\times \frac{1}{\left(r_{i}^{*}-r^{*}\right)^{2}}+\cdots=: \frac{v(k)^{2}-\frac{1}{4}}{\left(r_{i}^{*}-r^{*}\right)^{2}}+\cdots,
\end{gathered}
$$


$v(k)>0$. The subtleties mentioned above are the half-infinite domain for $r^{*}$ and the behaviour (29) of the potential at the $r_{i}^{*}$ boundary. These singular potentials are discussed in detail in [20]. The eigenfunctions of $\mathcal{H}$ which are square integrable for $r^{*}$ near $-\infty$ behave as in (18) in this limit and as

$$
\psi \sim a\left[\left(r_{i}^{*}-r^{*}\right)^{\frac{1}{2}+v}+\cdots\right]+b\left[\left(r_{i}^{*}-r^{*}\right)^{\frac{1}{2}-v}+\cdots\right]
$$

near the horizon, the $E$ eigenvalue appearing only in sub-leading terms. Thus, if $v>1$, for generic $E$ these are not square integrable near zero, unless $b=0$, and this is precisely the condition that selects a discrete set of possible $E$ values as the spectrum of $\mathcal{H}$, and that defines the space of functions where $\mathcal{H}$ is self-adjoint. On the other hand, if $v<1$, for any $E$ the eigenfunction behaving properly at minus infinity will be square integrable for $r^{*} \in(-\infty, 0)$, and a choice of boundary condition needs to be imposed to define a set of allowed perturbations $D_{\text {phys }}$, in order that $\mathcal{H}$ be a self-adjoint operator on $D_{\text {phys }}$ (and thus have a complete set of eigenfunctions) [21]. This situation, where a choice of self-adjoint extension needs to be done, is also found in the study of perturbations around the negative mass Schwarzschild spacetime [8], and perturbations in the inner region of a Reissner-Nordström spacetime [19]. Roughly, $D_{\text {phys }}$ is constructed by adding to the set of functions of compact support, those decaying as (30) with a fixed quotient $q:=b / a \in \mathbb{R}$, possibly infinite [21]. However, regardless of our choice for $q$, functions of compact support away from this singular point will belong to $D_{\text {phys }}$, in particular, the test function used in (19)-(20). This implies that the bound (21) will hold for any choice of boundary condition ( $q$ value)! In other words, region III of a sub-extreme Kerr black hole is unstable regardless of the boundary condition imposed on perturbations at the inner horizon. A natural choice would be that giving the fastest decay, i.e., $q=0$. In this case the gravitational perturbations behave as $\Phi \sim \Delta^{3 / 4}\left(r_{i}^{*}-r^{*}\right)^{\frac{1}{2}+v} \sim\left(r_{i}^{*}-r^{*}\right)^{2+v} \sim\left(r_{i}-r\right)^{1+v / 2}$. One should keep in mind that $v=v\left(k_{\ell}\right)$ is positive and grows for large $\ell$.

The instability found in region III of Kerr spacetime, and the fact that the ReissnerNordström charged black hole also has a two horizon structure with an inner static region $r<r_{i}$, triggers the question of whether or not this inner static region is stable. Preliminary work indicates that this region is unstable [19].

As a final comment, we note that our results do not contradict the established stability of the outer stationary, region I of Kerr black holes. For $a \leqslant M$ and $r>r_{o}$ we can define $r^{*}$ as in (11), and get again the Schrödinger form (13), but now the domain is restricted to $r_{o}<r$, where

$$
V>-\frac{15}{4} \quad \text { if } \quad a \leqslant M \text { and } 0<k .
$$

This implies that $\epsilon_{o}(k)<15 / 4$ for all $k>0$ and thus cannot intersect $\left.E_{\ell}(a \omega)\right|_{a \omega=\mathrm{i} k}$ for any $\ell$. To get the bound (31) is easy in the extreme case, for which (15) reduces to (replace $r=M r^{\prime}$, then drop the primes)

$$
V_{\text {ext }}=\left[\frac{r(r+1)\left(r^{2}-r+2\right)}{(r-1)^{2}}\right] k^{2}-4\left[\frac{r^{2}-2 r-1}{r-1}\right] k+\frac{1}{4}
$$

We search for critical points at fixed $k$ by solving $\partial V / \partial r=0$. This gives

$$
k=\frac{2(r-1)\left(r^{2}-2 r+3\right)}{\left(r^{2}+1\right)\left(r^{2}-2 r-1\right)},
$$

which has a unique $r>1=r_{i}=r_{o}$ solution for every positive $k$, with $r$ moving from infinity down to $1+\sqrt{2}$ as $k$ goes from zero to infinity. Since $\partial^{2} V / \partial r^{2}>0$ at these points, these are (global) minima of $V$ for fixed $k$. Moving along this curve from $r=1+\sqrt{2}$ to infinity, $V$ decreases monotonically to $-15 / 4$. In the sub-extreme case $a<M$ the equation $\partial V / \partial r=0$ is quadratic in $k$, and yields two curves like that in (33). However, one of these curves gives 
$k<0$ for all $r>r_{o}$, and should be discarded. The other one corresponds to minima of $V$ for fixed positive $k$, with $V$ again decreasing monotonically to $-15 / 4$ along it.

\section{Acknowledgments}

We thank Vitor Cardoso and Marc Casals for clarification of a number of issues related to prolate SWSHs. This work was supported in part by grants from CONICET (Argentina) and Universidad Nacional de Córdoba. RJG and GD are supported by CONICET. IFRS is a fellow of the CICBA.

\section{References}

[1] Kerr R P 1963 Phys. Rev. Lett. 11237

[2] Carter B 1997 Has the black hole equilibrium problem been solved? Proc. 8th Marcel Grossmann Meeting on Recent Developments in Theoretical and Experimental General Relativity, Gravitation and Relativistic Field Theories (Jerusalem, Israel, 22-27 June 1997) ed T Piran (Singapore: World Scientific) pp 136-55 (arXiv:gr-qc/9712038)

[3] Teukolsky S A 1972 Phys. Rev. Lett. 291114

Teukolsky S A 1973 Astrophys. J. 185635

[4] Whiting B F 1989 J. Math. Phys. 301301

[5] Poisson E and Israel W 1990 Phys. Rev. D 411796

[6] Dotti G, Gleiser R J and Pullin J 2007 Phys. Lett. B 644 289-93 (arXiv:gr-qc/0607052)

[7] Gleiser R J and Dotti G 2006 Class. Quantum Grav. 235063 (arXiv:gr-qc/0604021)

[8] Gibbons G W, Hartnoll D and Ishibashi A 2005 Prog. Theor. Phys. 113 963-78 (arXiv:hep-th/0409307)

[9] Cardoso V and Cavaglia M 2006 Phys. Rev. D 74024027 (arXiv:gr-qc/0604101)

[10] Leaver E W 1985 Proc. R. Soc. A 402285

[11] Seidel E 1989 Class. Quantum Grav. 61057

[12] Berti E, Cardoso V and Casals M 2006 Phys. Rev. D 73024013

Berti E, Cardoso V and Casals M 2006 Phys. Rev. D 73109902 (arXiv:gr-qc/0511111) (erratum)

[13] Kegeles L and Cohen J 1979 Phys. Rev. D 191641

Chrzanowski P 1975 Phys. Rev. D 112042

Wald R 1978 Phys. Rev. Lett. 41203

[14] See, e.g. Khanna G 2002 Phys. Rev. D 66064004

[15] Breuer R A 1975 Gravitational Perturbation Theory and Synchrotron Radiation (Lecture Notes in Physics vol 44) (Berlin: Springer)

[16] Breuer R A, Ryan M P Jr and Waller S 1977 Proc. R. Soc. A 35871

[17] Berti E, Cardoso V and Yoshida S 2004 Phys. Rev. D 69124018 (arXiv:gr-qc/0401052)

[18] Meixner J and Schäfke FW 1954 Mathieusche Funktionen und Sphäroidfunktionen (Berlin: Springer) Meixner J and Schäfke FW 1980 Mathieu Functions and Spheroidal Functions and their Mathematical Foundations: Further Studies (Lectures Notes in Mathematics vol 837) (Berlin: Springer)

[19] >Dotti et al (work in progress)

[20] Meetz K 1964 Il Nuovo Cimento 34690

[21] Reed M and Simon B Methods of Modern Mathematical Physics II: Fourier Analysis, Self-Adjointness (New York: Academic) section X.1 